\title{
ARTICULO ESPECIAL
}

\section{Enfoque de la hipertensión pulmonar en el paciente pediátrico}

\section{Lina María Caicedo-Cuenca*}

\author{
Fundación Clínica Shaio, Bogotá, Colombia
}

Recibido el 28 de octubre de 2016; aceptado el 5 de julio de 2017

Disponible en Internet el 8 de septiembre de 2017

\section{PALABRAS CLAVE}

Hipertensión arterial pulmonar;

Pediatría;

Niño;

Defectos cardiacos

congénitos

\section{KEYWORDS}

Pulmonary arterial

hypertension;

Pediatrics;

Child;

Congenital heart

defects

\begin{abstract}
Resumen Si bien la enfermedad vascular pulmonar hipertensiva en niños comparte similitudes con la de los adultos, difiere de manera relevante en cuanto a fisiopatología, presentación y tratamientos, ya que se ven involucrados aspectos del desarrollo, perinatales y etiologías múltiples. Solo recientemente se dispone de guías y consensos específicos que se discuten en este artículo, la mayoría con menor nivel de evidencia que los de adultos, como es usual en la edad pediátrica. Se presentan algoritmos diagnósticos específicos para el grupo etario, dadas las patologías más frecuentes, con énfasis en la importancia del cateterismo cardiaco idealmente como último estudio diagnóstico. El tratamiento se enfoca según el grupo etiológico, pero, a diferencia del de los adultos, el flujograma no se establece por clase funcional sino por escala de riesgo.

( 2017 Publicado por Elsevier España, S.L.U. en nombre de Sociedad Colombiana de Cardiología y Cirugía Cardiovascular. Este es un artículo Open Access bajo la licencia CC BY-NC-ND (http: / / creativecommons.org/licenses/by-nc-nd/4.0/).
\end{abstract}

\section{Approach to pulmonary hypertension in the paediatric patient}

Abstract Although hypertensive pulmonary vascular disease in children shares similarities with that of adults, it differs significantly as regards pathophysiology, presentation, since developmental, perinatal, and multiple aetiological aspects are involved. It is only recently that specific guidelines and consensuses have become available, which are discussed in this article, with the majority with a lower level of evidence than those of adults, as is usual in paediatrics. Specific diagnostic algorithms are present for the age group, given the most frequent diseases, with emphasis on the importance of cardiac catheterisation, ideally as the ultimate diagnostic study. The treatment is approached according to aetiological group, but unlike that of adults, the flowchart is not established by functional class but by a risk scale.

( $) 2017$ Published by Elsevier España, S.L.U. on behalf of Sociedad Colombiana de Cardiología y Cirugía Cardiovascular. This is an open access article under the CC BY-NC-ND license (http:// creativecommons.org/licenses/by-nc-nd/4.0/).

\footnotetext{
* Autor para correspondencia.

Correo electrónico: lina.caicedo@shaio.org
} 


\section{Introducción}

La hipertensión pulmonar no es una enfermedad sino una condición hemodinámica que puede estar asociada a diferentes patologías y que, en la mayoría de los casos, es progresiva y limitante, y además causa gran morbilidad y mortalidad asociada. En los niños clásicamente se considera como una entidad infrecuente, cuya incidencia y prevalencia son subestimadas.

Aunque existen más similitudes que diferencias con los adultos, los niños tienen diferente estructura y funcionamiento vascular, función del ventrículo derecho, clasificación funcional, fenotipo, evolución y respuesta a terapias. En los niños se involucran muchos aspectos del desarrollo y crecimiento pulmonar e influencias perinatales, y hay mayor número de etiologías múltiples en un mismo paciente como sucede en síndromes genéticos como el de Down ${ }^{1,2}$.

A diferencia de la amplia literatura en adultos, la de los niños es limitada. Hasta hace poco, la aproximación al estudio y manejo de los pacientes pediátricos con hipertensión pulmonar se basaba en la información de adultos y artículos pediátricos aislados, pero en los últimos años han publicado guías consenso de expertos avaladas por sociedades científicas reconocidas, basadas en revisión exhaustiva de la literatura pediátrica aunque aún sin el número de estudios y nivel de evidencia para muchas aproximaciones.

\section{Definición}

Se define hipertensión pulmonar como un valor de presión pulmonar media mayor de $25 \mathrm{~mm} \mathrm{Hg}$ en niños mayores de 3 meses y que habiten a nivel del $\operatorname{mar}^{3,4}$. Se cuestiona si este valor es el mismo a mayores alturas, pero no hay en el momento una definición establecida para cada una de estas.

En niños menores, prematuros y los de muy bajo peso, no existe una definición establecida y la aplicación de la anterior es debatible ya que es frecuente observar presión sistémica media $\leq 70 \mathrm{~mm} \mathrm{Hg}$, de modo que en la práctica clínica muchos médicos consideran más apropiado definir hipertensión pulmonar en estos pacientes con base en la proporción de la presión pulmonar media respecto a la presión sistémica media o con base en la presión pulmonar sistólica respecto a la presión sistémica sistólica mayor a 0,4 en cualquiera de las dos situaciones ${ }^{1}$.

El término enfermedad vascular pulmonar hipertensiva se usa con frecuencia como un término más amplio que incluye alteraciones en cuanto a tono, reactividad, crecimiento y estructura del lecho vascular pulmonar aún con presiones menores a $25 \mathrm{~mm}$ Hg como sucede en los pacientes con cirugía de Fontan y fisiología de ventrículo único ${ }^{5}$. Existe consenso en continuar la definición de resistencias pulmonares aumentadas cuando sea $\geq 3 \mathrm{UW} / \mathrm{m}^{2}$, tanto en niños como en adultos ${ }^{3,4,6}$. Igualmente, hay acuerdo en la definición posterior a anastomosis cavo-pulmonar como un índice de resistencia vascular pulmonar $\geq 3 \mathrm{UW} / \mathrm{m}^{2}$ o gradiente transpulmonar $\geq 6 \mathrm{~mm} \mathrm{Hg}^{3}$.
Tabla 1 Definiciones

Hipertensión pulmonar

PmAP > $25 \mathrm{~mm}$ Hg en niños $>3$ meses de edad a nivel del mar

Hipertensión arterial pulmonar

PmAP $>25 \mathrm{~mm} \mathrm{Hg}$

PEAP $<15 \mathrm{~mm} \mathrm{Hg}$

IRVP > WU/ $\mathrm{m}^{2}$

Hipertensión arterial idiopática o hipertensión arterial

pulmonar aislada

Hipertensión arterial pulmonar sin enfermedad

subyacente conocida

Referida como HPAH con historia familiar positiva o evaluación genética

Enfermedad vascular pulmonar hipertensiva

Categoría amplia que incluye formas de HAP, pero agrupa sujetos con gradiente transpulmonar elevado $=(\mathrm{PmAP}-$ presión auricular izquierda o PEAP $>6 \mathrm{~mm} \mathrm{Hg}$ ) o alta IRVP como se observa en pacientes con anastomosis cavo-pulmonares sin PmAP alta

HPAH: hipertensión arterial pulmonar hereditaria; HAPI: hipertensión arterial pulmonar idiopática; PmAP: presión media de la arteria pulmonar; HAP: hipertensión arterial pulmonar; HP: hipertensión pulmonar; EVPH: enfermedad vascular hipertensiva pulmonar; IRVD: índice de resistencia vascular pulmonar ${ }^{3}$.

En la tabla 1 se resumen las definiciones principales.

\section{Etiología y clasificación}

En la población pediátrica la causa más frecuente de hipertensión arterial pulmonar es la asociada con cardiopatías congénitas, idiopática y hereditaria (casi el $80 \%$ en muchas series) y muchas veces de orden multifactorial ${ }^{7}$.

Las primeras clasificaciones se basaron en aspectos histológicos, pero en la actualidad se enfatizan además aspectos clínicos y fisiopatológicos. Hoy existen dos clasificaciones diagnósticas vigentes: la del Quinto Simposio Mundial de hipertensión pulmonar Niza 2013 para adultos y niños, y la exclusivamente pediátrica del Pulmonary Vascular Research Institute (PVRI) de Panamá 20113,4,8.

Para aplicar en forma sistemática y ordenada lo que que se ha aprendido en hipertensión pulmonar, se desarrolló y modificó una clasificación diagnóstica en los simposios mundiales de hipertensión pulmonar (WPHS). Fue creada inicialmente en 1998 en Evian (Francia) y ha sido modificada en reuniones posteriores, si bien hoy está vigente la del 2013 modificada en Niza, en la que se consideró que adultos y niños deben compartir la clasificación y se incluyeron características específicas de la enfermedad en la etapa pediátrica ${ }^{3,6,9,10}$. La clasificación establece cinco categorías en las que cada grupo comparte características hemodinámicas, patológicas y de manejo (tabla 2$)^{6,10}$.

La clasificación expandida modificada en Niza 2013 ahora incluye categorías diferentes para la hipertensión pulmonar persistente del recién nacido (HPPRN) en una categoría independiente por sus características pato-biológicas únicas y su alta posibilidad de resolver cardiopatías del corazón 
Tabla 2 Clasificación de hipertensión pulmonar, Niza 2013

Clasificación clínica de la hipertensión pulmonar, $5^{\circ}$ Congreso Mundial-Niza $2013^{10}$

Grupo 1. Hipertensión arterial pulmonar (HAP)

1.1. Idiopática (HAPi)

1.2. Hereditaria

1.2.1. BMPR2

1.2.2. Mutaciones en los genes ALK1, endoglin, ENG, SMAD9, CAV1, KCNK3

1.2.3. Desconocida

1.3. Inducida por drogas y toxinas (metanfetaminas)

1.4. Asociada con HAPA

1.4.1. Enfermedades del tejido conectivo

1.4.2. Infección por VIH

1.4.3. Hipertensión portal

1.4.4. Enfermedades cardíacas congénitas

1.4.5. Esquistosomiasis

1' Enfermedad venooclusiva pulmonar (EVOP) y/o hemangiomatosis capilar pulmonar (HCP).

1'" Hipertensión pulmonar persistente del recién nacido.

Grupo 2. Hipertensión pulmonar debida a enfermedad cardíaca izquierda

2.1. Disfunción sistólica

2.2. Disfunción diastólica

2.3. Enfermedad valvular

2.4. Cardiopatías congénitas o adquiridas que afectan la entrada o salida del ventrículo izquierdo.

Grupo 3. Hipertensión pulmonar asociada a enfermedades pulmonares y/o a hipoxemia

3.1. Enfermedad pulmonar obstructiva crónica (EPOC)

3.2. Enfermedad pulmonar intersticial

3.3. Otras enfermedades pulmonares con patrón mixto restrictivo y obstructivo

3.4. Síndrome de apnea del sueño

3.5. Hipoventilación alveolar

3.6. Exposición crónica a grandes alturas

3.7. Displasias pulmonares

Grupo 4.Hipertensión pulmonar por enfermedad tromboembólica crónica (HPTEC)

Grupo 5. Hipertensión pulmonar con mecanismos multifactoriales no claros

5.1. Desórdenes hematológicos: Anemia hemolítica crónica, trastornos mieloproliferativos, esplenectomía

5.2. Trastornos sistémicos: sarcoidosis, histiocitosis de células de Langerhans: linfangioleiomiomatosis, vasculitis. 5.3. Trastornos metabólicos: enfermedades por depósito de glucógeno, enfermedad de Gaucher, desórdenes tiroideos. 5.4. Otros: obstrucción tumoral, mediastinitis fibrosante, insuficiencia renal crónica en diálisis, hipertensión pulmonar segmental.
Tabla 3 Clasificación hipertensión pulmonar pediátrica PVRI 20113

Las diez categorías básicas de la enfermedad vascular hipertensiva en Pediatría - PVRI - Consenso Panamá 2011.

1. Enfermedad vascular pulmonar hipertensiva prenatal o del desarrollo.

2. Maladaptación vascular pulmonar perinatal.

3. Enfermedad cardiovascular pediátrica.

4. Displasia broncopulmonar.

5. Enfermedad vascular pulmonar hipertensiva pediátrica aislada (HAP aislada).

6. Enfermedad vascular pulmonar hipertensiva multifactorial asociada a malformaciones y/o síndromes.

7. Enfermedad pulmonar

8. Enfermedad tromboembólica pediátrica.

9. Exposición del niño a la hipoxia hipobárica.

10. Enfermedad vascular pulmonar hipertensiva del niño asociada con alteraciones de otros sistemas.

izquierdo y miocardiopatías genéticas con genes recientemente identificados. La etiología de anemia hemolítica fue clasificada en el grupo 5 y la hipertensión pulmonar segmentaria se incorporó a un subtipo del grupo 5 .

El Task force realizó cambios a la clasificación de hipertensión pulmonar asociada a cardiopatías congénitas y reconoció la posibilidad de enfermedad vascular pulmonar en pacientes con correcciones univentriculares ${ }^{3,10,11}$.

En el año 2011, el PVRI propuso una clasificación específica extensa para la edad pediátrica, en la que se hace énfasis en los conceptos de maladaptación perinatal, alteraciones del desarrollo e hipoplasia pulmonar como factores causantes de la hipertensión pulmonar pediátrica, así como en la importancia de los múltiples síndromes malformativos congénitos, genéticos y cromosómicos. Con base en dicha clasificación, la enfermedad vascular pulmonar hipertensiva en niños se divide en diez grandes categorías (tabla 3$)^{5}$.

\section{Diagnóstico}

El diagnóstico inicial es tan importante como para cualquier enfermedad, así como lo son una historia clínica y un examen físico completos sumados a exámenes diagnósticos que permitan establecer la etiología/clasificación y el estado funcional del corazón. El proceso es escalonado; se parte de la sospecha clínica, requiere confirmación del diagnóstico e identificar la etiología específica (la hipertensión arterial pulmonar idiopática -HAPi- debe ser un diagnóstico de exclusión) y culmina con la evaluación de la gravedad.

La radiografía de tórax, el electrocardiograma y el ecocardiograma forman parte fundamental de la aproximación inicial. Este último es el examen de elección para tamizaje, y es útil además para evaluar y descartar algunas causas, así como para valorar la función del ventrículo derecho y algunos factores pronósticos en el seguimiento no invasivo $^{12-15}$.

La aproximación diagnóstica y el manejo requieren el conocimiento de la enfermedad y un enfoque sistemático, 


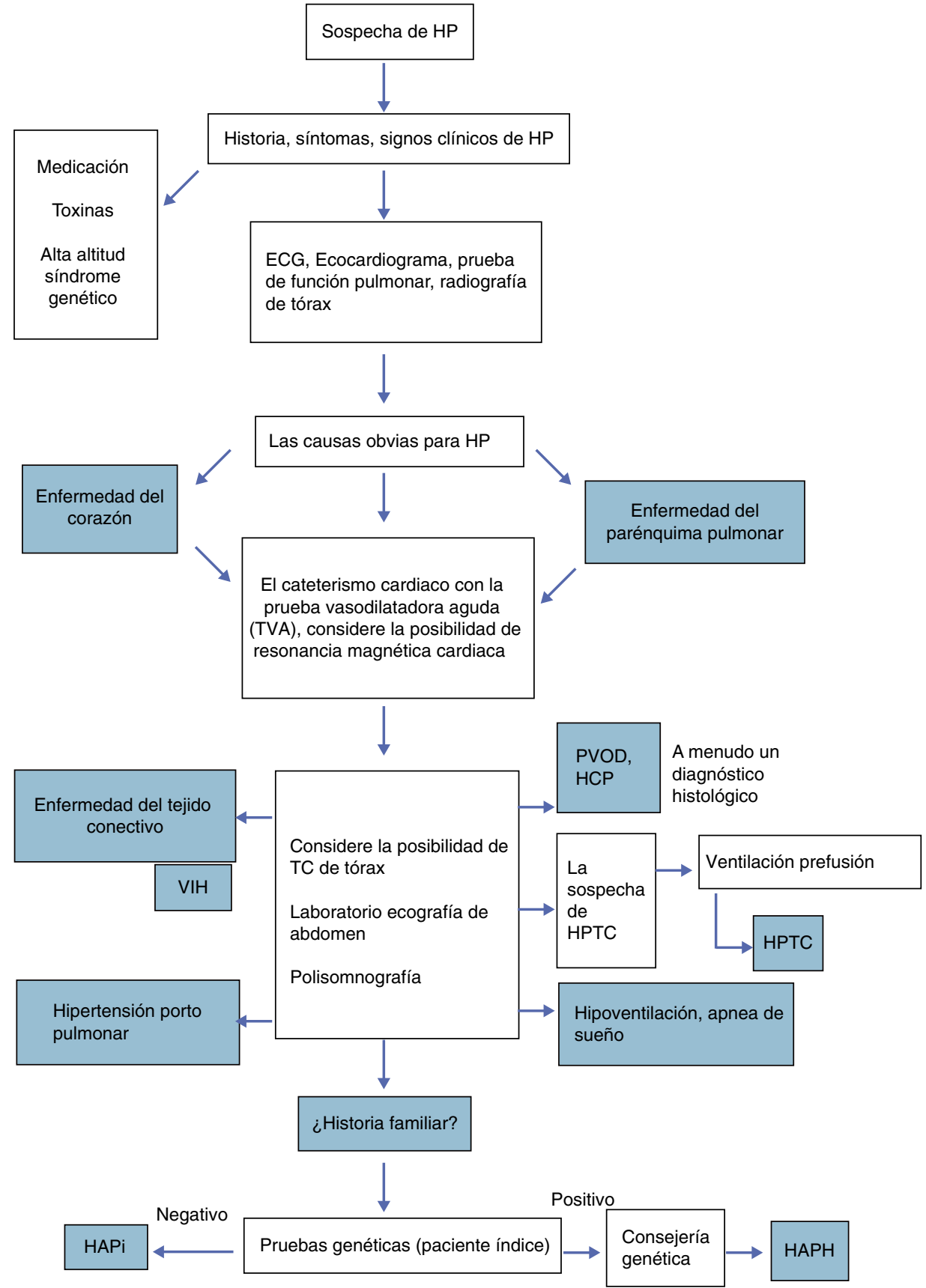

Figura 1 Flujograma diagnóstico ante sospecha de hipertensión pulmonar. CHD: enfermedad cardíaca congénita; HPTC: hipertensión pulmonar tromboembolia crónica; HAPH: HP hereditaria; HAPi: HAP idiopática; HCP: hemangiomatosis capilar pulmonar; HP: hipertensión pulmonar; PVOD: enfermedad veno-oclusiva pulmonar. Traducido de Lammers et $\mathrm{al}^{4}$.

de modo que dada la complejidad de la entidad y la importancia de la experiencia en los procedimientos diagnósticos y estrategias terapéuticas, se sugiere remitir a los pacientes a centros expertos de referencia, si bien los pasos iniciales y el tamizaje deben ser conocidos y aplicados por todos los médicos.

La aproximación que se muestra en la figura 1 se basa en las recomendaciones de consenso de las Sociedades europeas y americanas para esta enfermedad y en la búsqueda inicial de las causas más frecuentes con test adicionales en casos seleccionados o poblaciones de riesgo específico ${ }^{3,4}$.

\section{Cateterismo cardiaco}

Sigue siendo el estándar de oro para establecer el diagnóstico pero también tiene gran utilidad para determinar causas, manejo, estratificación de riesgo y respuesta al tratamiento.

En general, la evaluación invasiva hemodinámica ha de ser el último procedimiento diagnóstico, pero el cateterismo diagnóstico con test de reactividad, se realizará al menos una vez en los pacientes con enfermedad vascular pulmonar importante, a excepción de las formas transitorias y los 
Tabla 4 Medicamentos usados para TVA en Pediatría. Traducido de Consenso PVRI $2016^{16}$

\begin{tabular}{ll}
\hline & Cateterismo cardiaco - test de vasorreactividad \\
\hline Medicamento & Dosis informada \\
\hline Óxido nítrico inhalado & $20-80 \mathrm{ppm}$ \\
Iloprost inhalado & $0,5 \mu \mathrm{g} / \mathrm{kg}$ para pacientes $<15 \mathrm{~kg} ; 5 \mu \mathrm{g}$ para pacientes $>15 \mathrm{~kg}$ \\
Prostaciclina inhalada & $5-50 \mathrm{ng} / \mathrm{kg} / \mathrm{min}$ \\
Treprostinil inhalado & $1,53 \mu \mathrm{g} / \mathrm{kg}(\mathrm{rango}: 0,71-2,89)$ \\
Milrinone inhalado & $50 \mu \mathrm{g} / \mathrm{kg}$ \\
Nitroglicerina inhalada & $50 \mu \mathrm{gg}$ \\
Prostaciclina intravenosa & $2-10 \mathrm{ng} / \mathrm{kg} / \mathrm{min}$ ajuste de dosis: $2 \mathrm{ng} / \mathrm{kg} / \mathrm{min}$ cada $10-15 \mathrm{~min}$ \\
Adenosina intravenosa & $50-250 \mu \mathrm{g} / \mathrm{kg} / \mathrm{min}$ ajuste de dosis $50 \mu \mathrm{g} / \mathrm{kg} / \mathrm{min}$ cada $2 \mathrm{~min}$ \\
\hline
\end{tabular}

A. Tanto nitroglicerina inhalada como milrinone se preparan mezclando el fármaco con solución salina normal para hacer un volumen de $3 \mathrm{ml}$, y nebulizada durante 10 minutos con un nebulizador jet usando $8 \mathrm{~L} / \mathrm{min}$ de una mezcla de oxígeno-aire al 50\%

B. El uso de adenosina es controvertido y se carece de información acerca de su empleo en niños.

pacientes en estado crítico; debe revisarse en forma individual su indicación y riesgo en pacientes con síndrome de Eisenmenger establecido ${ }^{16}$. En forma ideal, debería realizarse en el paciente despierto pero tranquilo y colaborador, algo difícil en Pediatría, así que comúnmente se requiere anestesia general con respiración espontánea o sedación. La tasa de complicaciones serias va del 2 al $6 \%$ y la mortalidad del 0 al $1,4 \%{ }^{4,6,7}$.

Por otra parte, el test de reactividad vascular pulmonar (TVA) evalúa la respuesta del lecho vascular pulmonar a vasodilatadores específicos (tabla 4). En muchas instituciones se hace con oxígeno al $100 \%$ pero existe controversia sobre su valor para predecir operabilidad. La combinación de oxígeno y óxido nítrico (ON) es de mayor utilidad que cada uno en forma independiente, pero se han empleado varios medicamentos (tabla 4) ${ }^{16}$.

A través del tiempo se han usado diferentes definiciones para considerar positivo un TVA. Hay dos definiciones e interpretación del TVA: una sirve para evaluar pronóstico e indicación de terapia específica y la otra para ver operabilidad en los pacientes con cardiopatía. En HAPi se utilizan dos definiciones: la disminución de al menos $10 \mathrm{~mm} \mathrm{Hg}$ con valores menores de $40 \mathrm{~mm} \mathrm{Hg}$ con aumento o sin cambio en gasto cardiaco, y la diminución > del $20 \%$ de la presión media de la arteria pulmonar con aumento o sin cambio en el gasto cardiaco y disminución o sin cambio en la relación de resistencias IRVP/IRVS 3 . En cuanto al uso de calcioantagonistas se recomienda tomar como respondedores agudos para niños con enfermedad vascular pulmonar hipertensiva idiopática, los criterios estrictos de casi normalización de Sitbon, con disminución de IRVP a $<4,5 \mathrm{UW} / \mathrm{m}^{2}$ como lo indica Moledina ${ }^{16}$.

El cambio hemodinámico que define un respondedor agudo en cardiopatías con cortocircuitos simples es una caída mayor al $20 \%$ en el índice de resistencia vascular pulmonar (IRVP) y la relación de resistencias vasculares pulmonares y sistémicas (IRVP/IRVS) con valores menores a 6 $\mathrm{UW} / \mathrm{m}^{2} \mathrm{y}<0,3$ respectivamente ${ }^{8}$.

Tabla 5 Determinantes de riesgo en enfermedad vascular pulmonar pediátrica

\begin{tabular}{|c|c|c|}
\hline Menor riesgo & Determinantes de riesgo & Mayor riesgo \\
\hline No & $\begin{array}{l}\text { Evidencia clínica de } \\
\text { insuficiencia del ventrículo } \\
\text { derecho (VD) }\end{array}$ & $\mathrm{Si}$ \\
\hline No & Progresión de los síntomas & $\mathrm{Si}$ \\
\hline No & $\begin{array}{l}\text { Síncope } \\
\text { Crecimiento }\end{array}$ & $\mathrm{Si}$ \\
\hline I,II & Clase funcional & III, IV \\
\hline Mínimamente elevado & BNP/Pro BNP sérico & $\begin{array}{l}\text { Aumento de nivel significativamente } \\
\text { elevado }\end{array}$ \\
\hline & Ecocardiografía & $\begin{array}{l}\text { Crecimiento severo del } \\
\text { VD } \\
\text { VD disfunción } \\
\text { Derrame pericárdico }\end{array}$ \\
\hline $\begin{array}{l}\text { IC }>3,01 / \mathrm{min} / \mathrm{m}^{2} \\
\text { PmAp } / \mathrm{Pm} \mathrm{AS}<0,5 \\
\text { Reactividad vascular aguda }\end{array}$ & Hemodinámica & $\begin{array}{l}\mathrm{IC}<2,5 \mathrm{~L} / \mathrm{min} / \mathrm{m}^{2} \\
\mathrm{PmAP} / \mathrm{PmAS}>0,75 \\
\mathrm{PmAD}>15 \mathrm{~mm} \mathrm{Hg} \\
\mathrm{IRVP}>15 \mathrm{UW} \times \mathrm{m}^{2}\end{array}$ \\
\hline
\end{tabular}




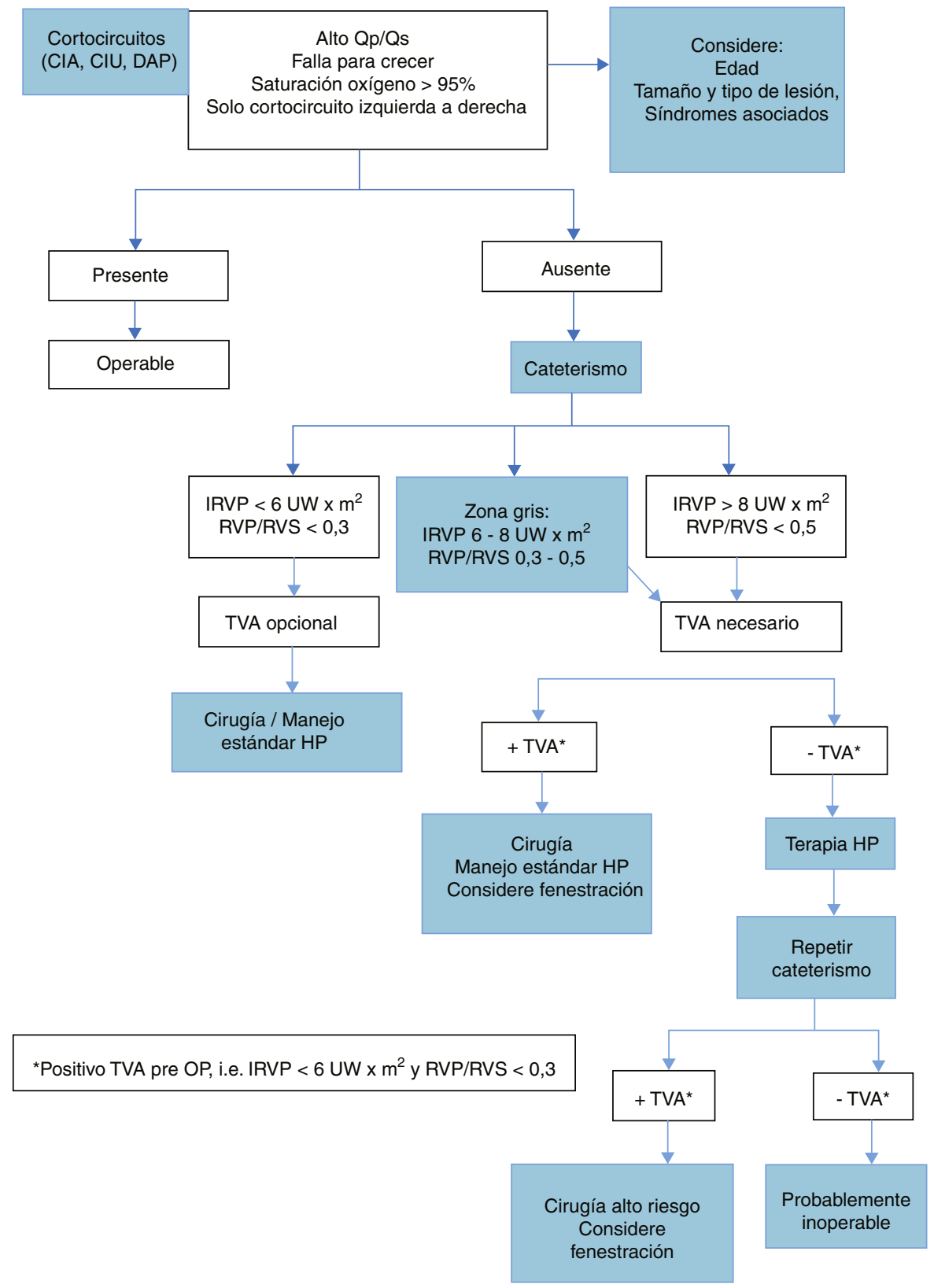

Figura 2 Flujograma de manejo en hipertensión arterial pulmonar asociada a cardiopatía congénita con cortocircuito. Traducido de Kozlik-Feldmann et $\mathrm{al}^{20}$.

\section{Hipertensión pulmonar y cardiopatías congénitas}

La hipertensión pulmonar más frecuente en niños es la asociada a cardiopatías congénitas, la mayoría con cortocircuitos pre- y postricúspides (perteneciente al grupo 1) pero también con enfermedades del corazón izquierdo (grupo 2) o la segmentaria (grupo 5).

La hipertensión pulmonar se puede presentar con o sin enfermedad vascular pulmonar hipertensiva y es importante establecer la diferencia para definir manejo quirúrgico. La evaluación y el tratamiento de la hipertensión pulmonar asociada a cardiopatías congénitas o enfermedad vascular pulmonar hipertensiva asociada a cardiopatías congénitas tiene varios aspectos por analizar; uno de ellos son los criterios de operabilidad e inicio de terapias avanzadas pre- y poscirugía correctora, tema aún no resuelto por completo $^{17}$.

En cardiopatías congénitas es importante enfatizar que la clave no está en el nivel de presión pulmonar sino en las resistencias vasculares pulmonares. La corrección temprana es crucial para evitar el remodelamiento de la vasculatura 
Tabla 6 Medicamentos más usados en hipertensión pulmonar infantil

\begin{tabular}{|c|c|}
\hline Agente & Dosificación \\
\hline Nifedipino & $\begin{array}{l}\text { Dosis inicial: } 0,1-0,2 / \mathrm{kg} \text { por vía oral } 3 \text { veces al día. Rango de } \\
\text { dosis: } 2-3 \mathrm{mg} / \mathrm{kg} / \text { día oral } \\
\text { Dosis para adultos máxima: } 180 \mathrm{mg} / \mathrm{dl} \text { por vía oral. }\end{array}$ \\
\hline Diltiazem & $\begin{array}{l}\text { Dosis inicial: } 0,5 \mathrm{mg} / \mathrm{kg} \text { por vía oral } 3 \text { veces al día. Rango de } \\
\text { dosis: } 3-5 \mathrm{mg} / \mathrm{kg} / \mathrm{día} \text { vía oral } \\
\text { Máxima dosis para adultos: } 360 \mathrm{mg} / \mathrm{dl} \text { por vía oral. }\end{array}$ \\
\hline Amlodipino & $\begin{array}{l}\text { Dosis inicial: } 0,1-0,3 \mathrm{mg} / \mathrm{kg} / \text { día vía oral. Rango de dosis: } 2,5 \\
-7,5 \mathrm{mg} / \text { día vía oral }\end{array}$ \\
\hline Sildenafil & $\begin{array}{l}\text { Edad <1 año: } 0,5-1 \mathrm{mg} / \mathrm{kg} 3 \text { veces al día por vía oral. } \\
\text { Peso < } 20 \mathrm{~kg}: 10 \mathrm{mg} 3 \text { veces al día por vía oral } \\
\text { Peso > } 20 \mathrm{~kg}: 20 \mathrm{mg} 3 \text { veces al día por vía oral } \\
\text { Retrasar su uso en prematuros extremos hasta que se } \\
\text { establezca la vascularización retiniana. }\end{array}$ \\
\hline Tadalafil & $\begin{array}{l}\text { Dosis inicial: } 0,5-1 \mathrm{mg} / \mathrm{kg} / \text { día } \\
\text { Máxima dosis: } 40 \mathrm{mg} \text { por vía oral día. } \\
\text { Evaluado sólo en los niños > } 3 \text { años de edad. }\end{array}$ \\
\hline Bosentan (antagonista dual ET A y ET B) & $\begin{array}{l}\text { La dosis inicial es la mitad de la dosis de mantenimiento } \\
\text { Dosis de mantenimiento: } \\
\text { Peso > } 10 \mathrm{~kg}: 2 \mathrm{mg} / \mathrm{kg} \text { dos veces al día por vía oral. } \\
\text { Peso } 10-20 \mathrm{~kg} 31,25 \mathrm{mg} \text { dos veces al día } \\
\text { Peso > } 20-40 \mathrm{~kg}: 62,5 \mathrm{mg} \text { dos veces al día } \\
\text { Peso > } 40 \mathrm{~kg}: 125 \mathrm{mg} \text { dos veces al día. }\end{array}$ \\
\hline Ambrisentan (un antagonista de ET A altamente selectivo) & $\begin{array}{l}\text { Rango de dosis: } 5 \text { - } 10 \mathrm{mg} \text { al día por vía oral } \\
\text { Uso en pacientes pediátricos }<5 \text { años de edad no estudiado. }\end{array}$ \\
\hline Iloprost (inhalación intermitente) & $\begin{array}{l}\text { Las dosis pediátricas no se han determinado pero se necesitan } \\
6-9 \text { inhalaciones por día, con una duración de } 10 \text { - } 15 \text { minutos } \\
\text { Comenzar con dosis de } 2,5 \mu \mathrm{g} \text { y aumentar hasta la dosis de } \\
5 \mu \mathrm{g} \text { según la tolerancia }\end{array}$ \\
\hline
\end{tabular}

pulmonar. Pacientes con cortocircuitos simples, en primeros meses de la vida, con saturación normal y signos de falla cardiaca, en general pueden ser operados sin necesidad de cateterismo cardiaco y TVA $^{18,19}$.

La figura 2 constituye el flujograma para decidir el manejo de pacientes con hipertensión pulmonar asociada a cardiopatía congénita sugerido por las últimas guías de consenso ${ }^{20}$. Se anotan en la sección de cateterismo los criterios del TVA positivo aclarando que ningún valor ha probado ser contraindicación absoluta para cirugía ${ }^{19}$.

\section{Tratamiento y seguimiento}

Durante los últimos años ha habido importantes avances en el manejo, aunque en Pediatría el uso de muchas medicaciones vasodilatadoras pulmonares específicas es off-label o en forma compasiva, por ausencia de estudios suficientes en niños. El manejo consiste en la llamada terapia de soporte y medicaciones específicas. Es crucial identificar la causa de la hipertensión pulmonar para manejarla y/o identificar si se beneficia de terapias específicas. El tratamiento y seguimiento en niños, a diferencia del de los adultos, no se guía en forma preferencial por clase funcional, sino con base en determinantes de riesgo (tabla 5) ${ }^{3,8,21,22}$. El propósito fundamental es la transición del paciente de alto a bajo riesgo.

En la figura 3 se presentan las recomendaciones y el flujograma de manejo del consenso internacional para HAPi e HAPH, con dosis de medicamentos (tabla 6) (3,4 $^{3, \text { Cada vez }}$ más se sugiere terapia combinada temprana e intervenciones como la atrioseptostomía o la recientemente sugerida cirugía de Potts ${ }^{19}$. El seguimiento de rutina se hace al menos cada 3-6 meses o con visitas más frecuentes ante enfermedad avanzada o cambios en el manejo. A pesar del desarrollo terapéutico el pronóstico aún es pobre y parece serlo más en niños que en adultos. 


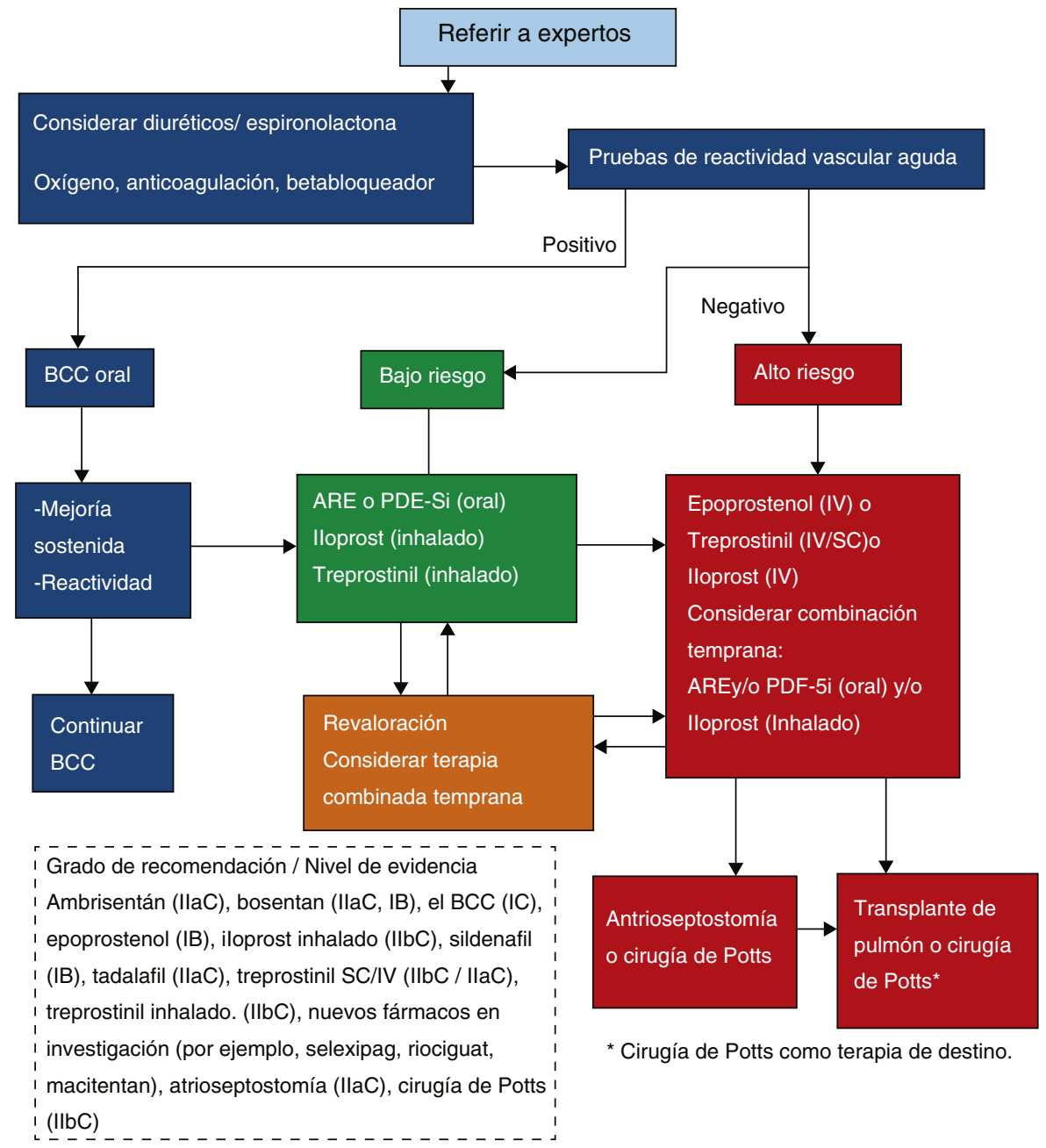

Figura 3 Flujograma de manejo. Traducido de Guías AHA $2015^{3}$.

\section{Conflictos de interés}

Ninguno.

\section{Bibliografía}

1. Barst RJ, Ertel SI, Beghetti M, et al. Pulmonary arterial hypertension: a comparison between children and adults. Eur Respir J. 2011;37:665-77.

2. Ambalavanan N, Mourani P. Pulmonary hypertension in bronchopulmonary dysplasia. Birth Defects Res A Clin Mol Teratol. 2014;100:240-6.

3. Abman SH, Hansmann G, Archer SL, et al. Pediatric pulmonary hypertension. Circulation. 2015;132:2037-99.

4. Lammers AE, Apitz C, Zartner P, et al. Diagnostics, monitoring and outpatient care in children with suspected pulmonary hypertension/paediatric pulmonary hypertensive vascular disease Expert consensus statement on the diagnosis and treatment of paediatric pulmonary hypertension. The European Paediatric Pulmonary Vascular Disease Network, endorsed by ISHLT and DGPK. Heart. 2016;102:ii1-13.

5. Cerro MJ, Del, Abman S, Diaz G, et al. A consensus approach to the classification of pediatric pulmonary hypertensive vascular disease: Report from the PVRI Pediatric Taskforce Panama 2011. Pulm Circ. 2011;1:286-98.
6. Galiè N, Humbert M, Vachiery J-L, et al. 2015 ESC/ERS Guidelines for the diagnosis and treatment of pulmonary hypertension. Eur Heart J. 2015;37:67-119.

7. Beghetti M, Berger RMF, Schulze-Neick I, et al. Diagnostic evaluation of paediatric pulmonary hypertension in current clinical practice. Eur Respir J [Internet]. 2013;42:689-700 [Acceso 18 Jun 2016] Disponible en: ht**tp://ow.ly/mx4GW.

8. Ivy DD, Abman SH, Barst RJ, et al. Pediatric pulmonary hypertension. J Am Coll Cardiol. 2013;62:D117-26.

9. Gali N, Simonneau G. The fifth world symposium on pulmonary hypertension. J Am Coll Cardiol. 2013;62:D1-3.

10. Simonneau G, Gatzoulis MA, Adatia I, et al. Updated Clinical Classification of Pulmonary Hypertension. J Am Coll Cardiol. 2013;62:D34-41.

11. Ivy DD, Abman SH, Barst RJ, et al. Pediatric Pulmonary hypertension. J Am Coll Cardiol. 2013;62(25.): D117-26.

12. Pansy J, Koestenberger M. Abnormalities in echocardiographic indices of right- and left- ventricular systolic function in pediatric pulmonary artery hypertension. Pediatr Cardiol. 2014:741-2.

13. Forfia PR, Vachiéry J. Echocardiography in pulmonary arterial hypertension. Am J Cardiol. 2012;110:S16-24.

14. Bossone E, Andrea AD, Alto MD, et al. Echocardiography in pulmonary arterial hypertension: from diagnosis to prognosis. J Am Soc Echocardiogr [. 2013;26:1-14. 
15. Vorhies EE, Gajarski RJ, Yu S, et al. Echocardiographic evaluation of ventricular function in children with pulmonary hypertension. Pediatr Cardiol. 2014:759-66.

16. del Cerro MJ, Moledina S, Haworth SG, et al. Cardiac catheterization in children with pulmonary hypertensive vascular disease: consensus statement from the Pulmonary Vascular Research Institute Pediatric and Congenital Heart Disease Task Forces. Pulm Circ. 2016;6:118-25.

17. Myers PO, Beghetti M, Strategy LS. Assessment of operability of patients with. Circ J. 2014;78:4-11.

18. Lopes AA, Barst RJ, Haworth SG, et al. Repair of congenital heart disease with associated pulmonary hypertension in children: what are the minimal investigative procedures? Consensus statement from the Congenital Heart Disease and Pediatric Task Forces, Pulmonary Vascular Research Institute (PVRI). Pulm Circ. 2014;4:330-41.

19. Hansmann G, Apitz C, Abdul-Khaliq H, et al. Executive summary Expert consensus statement on the diagnosis and treatment of paediatric pulmonary hypertension The European Paediatric Pulmonary Vascular Disease Network, endorsed by ISHLT and DGPK. Heart. 2016;102:ii86-100.

20. Kozlik-Feldmann R, Hansmann G, Bonnet D, et al. Pulmonary hypertension in children with congenital heart disease (PAH-CHD PPHVD-CHD). Expert consensus statement on the diagnosis and treatment of paediatric pulmonary hypertension The European Paediatric Pulmonary Vascular Disease Network, endorsed by ISHLT and DGPK. Heart. 2016;102: ii $42-8$.

21. Nicolarsen J, Ivy D. Progress in the diagnosis and management of pulmonary hypertension in children. Curr Opin Pediatr. 2014;26:527-35.

22. Chen I-C, Dai Z-K. Insight into pulmonary arterial hypertension associated with congenital heart disease (PAH-CHD): Classification and pharmacological management from a pediatric cardiological point of view. Acta Cardiol Sin. 2015;31: 507-15. 